duct of anaesthesia, rather than the method, which contributes to the successful maternal and fetal outcome.

B. Venkatesh MBBS MD FFARCSI FRCA

University Dept. of Anaesthesia and Intensive Care

Queen Elizabeth Hospital, Edgbastond

Birmingham, B15 2TH

United Kingdom

P.C.M. Taggart MBBS FRCA

Consultant Anaesthetist

George Eliot Hospital, Nuneaton

CV10 7DJ

United Kingdom

REFERENCE

1 Venkatesh B, Taggart PCM. Anaesthetic management of a patient with MoyaMoya disease for Caesarean section (Letter). Can J Anaesth 1994; 41: 79-80.

\section{Anaesthesia for adenotonsillectomy}

To the Editor:

Dr. Webster $e t$ al. are to be commended for examining the role of a prototype armoured laryngeal mask airway for anaesthesia for the adenotonsillectomy in children. ${ }^{1}$ I wish to raise some questions regarding their methods and results and the clinical context of the study.

The authors describe the four anaesthetists taking part in this study as "initially relatively unfamiliar with the LMA for T\&A." They also state that "with practice and the improved conditions obtained when the dose of propofol was increased in each group to $3.5-4.0 \mathrm{mg} \cdot \mathrm{kg}^{-1}$, a satisfactory airway was obtained using the LMA more rapidly with tracheal intubation." Thus, the experimental conditions were changed twice during the study: (1) The anaesthetists became more experienced and (2) the dose of propofol was increased. This may have led to bias against the LMA in the initial phase of the study and may explain "the difficulty in inserting the LMA in ten cases (18.2\%), compared with none during tracheal intubation $(P<0.001)$."

It is not surprising that the heart rate, systolic and mean blood pressure were higher in the ETT group than in the LMA group since all 54 patients in the first group received atropine but only 12 of 54 in the latter group were similarly given atropine. Moreover, the greater blood loss may be attributed to the higher blood pressure. The universal use of atropine in this age group may be a matter of debate, but one can also argue that the healthy children in this study would not have suffered harm if all had received atropine in order to standardize the anaesthetic. In addition, there was no attempt to standardize the surgical technique which may also have introduced bias in the assessment of blood loss.

Similarly, it is not surprising that different results were obtained from the two groups in response to handling of the airway. When the authors calculated the mean duration from $i v$ induction to insertion of the LMA or ETT, did they take into account the extra time required for giving atropine and succinylcholine? In addition, the time to jaw relaxation after propofol can be expected to be shorter than that required for onset of full neuromuscular blockade. The same can be said for the time to return of spontaneous respiration which must have been considerably longer in the ETT group who had been paralyzed. It is misleading to present results as mean and standard deviation when the data are not normally distributed.

Finally, I would like to interpret this study in a clinical context. For the purpose of this study the authors arbitrarily defined desaturation as $\mathrm{SpO}_{2} \leq 94 \%$. What prompted them to pick this number and does transitory desaturation below this point have any clinical relevance? The authors report that the ETT group had lower oxygen saturation on arrival in the recovery room (95.9 \pm 2.21 and $93.5 \pm 4.53, P<0.05$ ). What is the relevance of these numbers when the accuracy of most pulse oximeters is reported to be $\pm 2-3 \% .^{2}$ If the observed difference had caused any clinical concern why were the children not transported to the recovery room with oxygen? The authors quote the work of Patel et al. ${ }^{3}$ who state in their article "The clinical importance of transient saturation of less than $90 \%$ is not known."

The authors' definition of stridor "as a high-pitched laryngeal sound with inspiratory soft tissue and lower rib retraction, but no oxyhaemoglobin desaturation with assisted bag/mask ventilation with $100 \%$ oxygen" hardly deserves to be labelled a complication but rather describes an event which was promptly handled by a vigilant and competent anaesthetist. The same can probably be said for observing brief desaturation with inserting either the LMA or the ETT or with opening the Boyle-Davis gag. It is of note that the more unsettling occurrences of laryngospasm and desaturation $\leq 94 \%$ as reactions to removing the airway were fairly equally distributed between the two groups. Again, what are the clinical consequences of these observations? Did any child need hospitalization subsequent to these events or suffer any damage? Is it not reasonable to state that a certain number of children whose airways are manipulated by a number of different techniques and instruments will respond with breathholding, desaturation or laryngospasm? Observing a variation from a predefined normal state or alteration of a physiological variable does not create a complication per se but serves as a warning signal for a potentially serious 
outcome. It simply means that a competent anaesthetist should be present.

Unfortunately, this study does not answer the basic question of whether the LMA is a useful or good alternative to endotracheal intubation in children who undergo adenotonsillectomy.

Thomas Hackmann MD

IWK Children's Hospital

Halifax, NS

\section{REFERENCES}

1 Webster AC, Morley-Foster PK, Dain S, et al. Anaesthesia for adenotonsillectomy: a comparison between tracheal intubation and the armoured laryngeal mask airway. Can J Anaesth 1993; 40: 1171-7.

2 Taylor MB, Whitwam JG. The accuracy of pulse oximeters. A comparative clinical evaluation of five pulse oximeters. Anaesthesia 1988; 43: 229-32.

3 Patel RI, Hannallah RS, Norden J, Casey WF, Verghese $S T$. Emergence airway complications in children: a comparison of tracheal intubation in awake and deeply anesthetized patients. Anaesth Analg 1991; 73: 266-70.

\section{$R E P L Y$}

We appreciate Dr. Hackmann's analysis of our paper, and the opportunity to reply to his observations. Dr. Hackmann is quite correct in pointing out that the greater incidence of difficulty in inserting the LMA was due to our initial unfamiliarity with the technique. The dose of propofol recommended for induction of paediatric anaesthesia $\left(2.5 \mathrm{mg} \cdot \mathrm{kg}^{-1}\right)$ did not consistently provide adequate conditions for insertion of the LMA. Once we increased our induction dose, we experienced no further difficulty in positioning the airway. Similarly, the cause of airway obstruction seen after opening the B-D gag and surgical stimulation was not immediately apparent. When we realized that the probable cause was reflex laryngeal closure with inadequate depth of anaesthesia, the incidence of problems diminished. These difficulties did lead early to bias against the LMA but, rather than redesign the study, we reported our problems in order that others may benefit from our experience. No child needed hospitalization or suffered any damage from these or other adverse events.

It has been shown that insertion of the LMA in the adult produces less cardiovascular response than laryngoscopy and intubation.' It is reasonable to postulate that similar effects are seen in children. We acknowledge the possible contribution of atropine and succinylcholine to haemodynamic differences in our two study groups. One of the advantages of the LMA is that it is not necessary to give either drug.

The difference in blood loss in the two groups may be attributable not only to differences in systemic arterial pressure, but also in venous pressure. Positive pressure ventilation, during which central venous pressure is increased, was required more often in the tracheal intubation group.

The shorter interval from iv induction to establishment of an airway with the LMA is primarily due to the extra time required to wait for the action of succinylcholine. In the ET group there was wide variance in the interval before onset of spontaneous breathing, reflected in large SD's. Some patients required assisted ventilation throughout surgery. The KruskallWallis test used in the analysis is a non-parametric test, and does not assume that the data is normally distributed.

An $\mathrm{SpO}_{2} \leq 94 \%$ defines a desaturation threshold, intended to provide early warning of hypoxic events. Transitory desaturation below this point is unlikely to have clinical significance. We agree with Dr. Hackmann that the difference in oxyhaemoglobin saturation between groups on arrival in the PACU, reported as mean values and $S D$, is probably not clinically relevant.

The significance of stridor is that it may herald the more serious complication of laryngospasm. Although it can readily be handled by the alert anaesthetist, most of us would prefer to avoid it. Controversy continues concerning the optimum condition for tracheal extubation in children. We extubated the trachea and the LMA with the child sufficiently deeply anaesthetized to suppress airway reflexes. Incidents of upper airway obstruction before return of consciousness led to insertion of oropharyngeal airways, and further potential upper airway irritation.

A.C. Webster MB

P.K. Morley-Forster MD

S. Dain MD

S. Ganapathy MD

The University of Western Ontario

Department of Anaesthesia

St. Joseph's Health Centre

REFERENCE

1 Wilson IG, Fell D, Robinson SL, Smith G.

Cardiovascular responses to insertion of the laryngeal mask. Anaesthesia 1992; 47: 300-2.

\section{Postoperative recurrent paralysis in an infant after mivacurium infusion}

To the Editor:

We wish to report a healthy two-month-old, 6-kg girl who underwent repair of a congenital cleft lip. During general anaesthesia, a mivacurium continuous infusion was used for neuromuscular blockade and was monitored with a nerve stimulator. Fifty minutes after starting the infusion, $60 \mathrm{mg}$ mivacurium had been administered $(0.2$ $\left.\mathrm{mg} \cdot \mathrm{kg}^{-1} \cdot \mathrm{min}^{-1}\right)$. Because of concerns for volume overload, the infusion was discontinued and neuromuscular blockade was maintained with bolus dosing. Additional doses of $0.08,0.04$, and $0.04 \mathrm{mg} \cdot \mathrm{kg}^{-1}$ were given at approximately $30 \mathrm{~min}$ intervals with two to three twitches remaining after each dose. Approximately five minutes after the last dose was given, the surgical procedure ended and neostigmine, $0.07 \mathrm{mg} \cdot \mathrm{kg}^{-1}$, and atropine, 0.025 $\mathrm{mg} \cdot \mathrm{kg}^{-1}$ were administered. Reappearance of four twitches was confirmed and the trachea was extubated 